\title{
Was nützt Akupunktur in der Supportivtherapie?
}

\section{Zahlreiche Studien haben sich mit dem Wert der Akupunktur bei Krebspatienten beschäftigt. Aber ihre Qualität ist höchst unterschied- lich. Ein systematischer Review hilft, die Spreu vom Weizen zu trennen.}

V iele Krebspatienten nutzen komplementäre Verfahren wie Akupunktur. Aber helfen ihnen die Nadeln wirklich? In den USA schlossen die National Institutes of Health 1998, dass Akupunktur effektiv für die Kontrolle postoperativer Schmerzen ist und auch gegen Chemotherapie-induzierte Nausea und Vomitus hilft. Doch Akupunktur zur Symptomlinderung bei Krebspatienten ist nach wie vor umstritten. In einem systematischen Review wurden nun alle verfügbaren prospektiven randomisierten Studien ausgewertet. Es wurden nur Studien eingeschlossen, bei denen Nadeln auch in Akupunkturpunkte gesetzt wurden; die Sprache der Publikation war irrelevant.
Von 2.151 Publikationen erfüllten nur 41 alle Kriterien. Sie betrafen Akupunktur zur Behandlung von Schmerz, Nausea, Hitzewallungen, Fatigue, strahleninduzierter Xerostomie, verlängertempostoperativem Ileus, Ängstlichkeit/ Stimmungsstörungen und Schlafstörungen. Nur eine dieser Studien hatte ein niedriges Bias-Risiko nach den Cochrane-Kriterien. Sie bescheinigt der Akupunktur einen deutlich positiven Effekt auf Chemotherapie-induzierte Nausea und Vomitus. Acht Studien hatten ein unklares Bias-Risiko: vier gingen positiv für die Akupunktur aus, drei negativ und eine hatte ein unklares Ergebnis.

33 Studien hatten ein hohes Bias-Risiko: 19 waren positiv für die Akupunktur und elf negativ; die übrigen drei bescheinigten der Akupunktur - abhängig vom betrachteten Symptom - teilweise einen Nutzen, teilweise aber auch nicht.

Fazit: Akupunktur scheint als zusätzliche Option gegen Chemotherapie-induzierte Nausea und Vomitus nützlich zu sein;

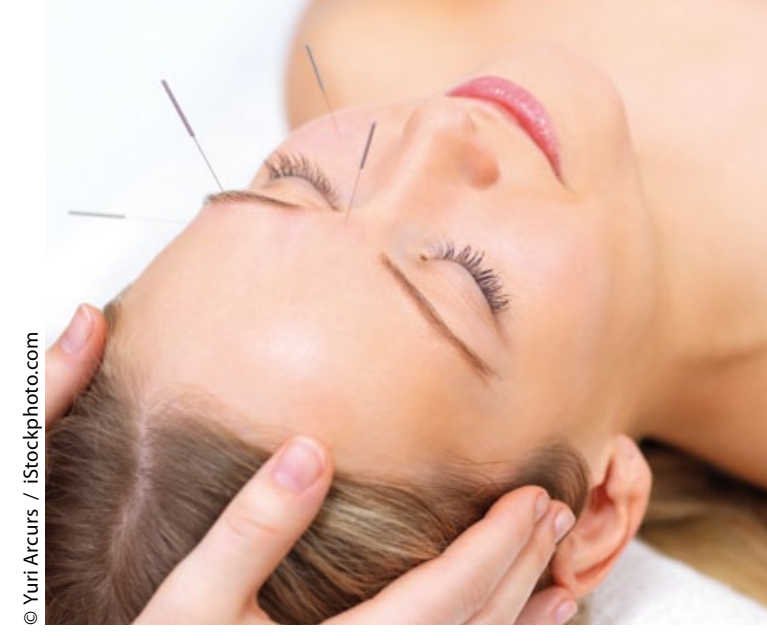

Soll segensreiche Wirkung haben, aber mit der Evidenz steht es noch nicht zum Besten: Akupunktur in der Onkologie.

aber es sind weitere Studien nötig, um das sicher zu belegen. Für andere Symptome ist eine Aussage wegen des hohen Bias in den Studien schwierig. Bei künftiger Forschung zu Akupunktur sollten Studien mindestens einfach verblindet sein, biologische Mechanismen untersuchen, eine adäquate statistische Aussagekraft haben und viele behandelnde Akupunkteure einbeziehen.

Christina Berndt

Garcia MK et al. Systematic review of acupuncture in cancer care: A synthesis of the evidence. J Clin Oncol. 2013;31(7):952-60.

\section{Psychosoziale Belastung von Krebspatienten mit minderjährigen Kindern}

\section{Der Einfluss der Elternschaft auf die psychosoziale Situation von Krebs- patienten wurde bisher kaum untersucht. Ein neu entwickelter, 15 Punk- te umfassender Fragebogen soll betreuungsbedürftige Krebspatienten mit minderjährigen Kindern identifizieren helfen.}

\footnotetext{
E pidemiologischen Untersuchungen zufolge haben $18 \%$ der erwachsenen Patienten mit der Erstdiagnose „Krebs“ minderjährige Kinder. Die gängigen Messinstrumente zur Einschätzung der Lebensqualität von Krebspatienten fokussieren meist nur auf generelle Aspekte zur krankheitsbedingten familiären Belastung und eher selten auf Kinder im betroffenen Haushalt. Auch existieren keine Publikationen über Testinstrumente zum Einfluss der elterlichen Krebserkrankung auf die Kinder.

Der elterliche Status beeinflusst in hohem Maß die Therapieentscheidung in frühen sowie in fortgeschrittenen Krankheitsstadien. Eltern von versor-
}

gungsbedürftigen Kindern leiden, wenn sie fortgeschritten an einem Malignom erkrankt sind, häufiger unter Panikstörungen und Angst als Kinderlose und sind eher bereit, eine aggressivere Therapie zu akzeptieren.

In dieser Phase-II-Studie wurde ein „Parenting Concerns Questionnaire“ (PCQ) für erwachsene Krebspatienten mit versorgungsbedürftigen Kindern erstellt. Als Basis diente die qualitative Auswertung von Gruppendiskussionen und quantitative Analysen. 173 in der Mehrheit weibliche onkologische Patienten mit Kindern unter 18 Jahren füllten einen zunächst 38 Punkte umfassenden Fragebogen $\mathrm{zu}$ „parental concerns“ aus.
Zudem kamen folgende psychoonkologische Screeningverfahren zum Einsatz: HADS (Hospitality Anxiety and Depression Scale), Distress Thermometer und FACT-G (Functional Assessment of Cancer Therapy-General). Die Analysen mündeten im neuen PCQ mit drei, jeweils fünf Punkte umfassenden SubItems, der in guter Übereinstimmung mit den bekannten Screening-Tests steht.

Fazit: Der neu entwickelte, 15 Punkte umfassende PCQ erwies sich als valides Messinstrument zur Beurteilung der psychosozialen Belastung und Betreuungsbedürftigkeit von erwachsenen Krebspatienten mit minderjährigen Kindern. Wolfgang Zimmermann

Muriel AC et al. Measuring psychosocial distress and parenting concerns among adults with cancer - the parenting concerns questionnaire. Cancer. 2012;118(22):5671-8. 